\title{
Correction to: An efficient pixel-level chaotic image encryption algorithm
}

\author{
Guodong Ye - Chen Pan - Xiaoling Huang • \\ Qixiang Mei
}

Published online: 31 October 2018

(C) Springer Nature B.V. 2018

\section{Correction to: Nonlinear Dyn}

$$
\text { https://doi.org/10.1007/s11071-018-4391-y }
$$

The correct affiliations are as follows:

Guodong Ye, Chen Pan, Xiaoling Huang, Qixiang Mei

Faculty of Mathematics and Computer Science, Guangdong
Ocean University, Zhanjiang 524088, China

e-mail: guodongye@ hotmail.com

Guodong Ye

College of Information Science and Electronic Engineering, Zhejiang University, Hangzhou 310027, China

The original article can be found online at https://doi.org/10. 1007/s11071-018-4391-y.

G. Ye $(\varangle) \cdot$ C. Pan $\cdot$ X. Huang $\cdot$ Q. Mei

Faculty of Mathematics and Computer Science,

Guangdong Ocean University, Zhanjiang 524088, China

e-mail: guodongye@hotmail.com

G. Ye

College of Information Science and Electronic Engineering,

Zhejiang University, Hangzhou 310027, China 